01

\title{
Определение равновесного состава термически ионизованного одноатомного газа в разных физических условиях
}

\author{
(C) М.C. Романова, М.А. Рыдалевская
}

Санкт-Петербургский государственный университет, 198504 Санкт-Петербург, Россия

e-mail: mariarom1990@mail.ru, rydalevska@rambler.ru

(Поступило в Редакцию 14 сентября 2016 г.)

Рассмотрены идеальные газовые смеси, полученные в результате термической ионизации пространственно и химически однородных одноатомных газов. Равновесные концентрации компонентов таких смесей определены путем интегрирования по пространству импульсов и суммирования по уровням энергии функций распределения, максимизирующих энтропию системы в условиях сохранения общего числа ядер и электронов. Показано, что такой метод позволяет не только значительно упростить расчет равновесного состава ионизованных смесей при разных температурах, но и исследовать зависимость степени ионизации газа от его плотности и номера в периодической системе элементов.

DOI: 10.21883/JTF.2017.05.44436.2034

\section{Введение}

Важным этапом решения многих научных и технологических задач является определение равновесного состава ионизованных газовых смесей. Проблеме исследования равновесных состояний плазмы всегда уделялось и продолжает уделяться большое внимание в энциклопедиях, монографиях и тематических сборниках (см., например, [1-7]). В принципе с общих позиций ее можно считать решенной. Однако система уравнений, которая решается в каждом конкретном случае, является достаточно сложной, так как включает нелинейные уравнения Саха. Коды, которые используются для решения этих систем, не всегда позволяют проследить влияние различных физических условий и особенностей исследуемого газа на степень его термической ионизации.

Для определения равновесного состава термически ионизованных одноатомных газов в настоящей работе используется метод, в основе которого лежит статистическое описание газовых систем. При этом равновесные концентрации атомов, ионов и свободных электронов определяются на основе наиболее вероятных функций распределения этих микрочастиц. Такой подход позволяет избежать решения уравнений Саха и свести определение равновесного состава ионизованного газа к решению одного алгебраического уравнения, а также исследовать влияние разных физических факторов на температурные зависимости равновесных концентраций всех микрочастиц исследуемой смеси.

\section{1. Равновесные функции распределения в ионизованном газе}

Для описания равновесных состояний изолированного объема идеального газа можно использовать функции распределения, максимизирующие энтропию систе- мы [8]. При квазиклассическом описании поступательных и квантовомеханическом внутренних степеней свободы микрочастиц равновесные функции распределения в изотропном пространственно-однородном газе могут быть представлены в виде [9]

$$
f_{c i}\left(\mathbf{p}_{c}\right)=\frac{s_{c i}}{h^{3}} \exp \left(\gamma_{0}\left(\frac{p_{c}^{2}}{2 m_{c}}+\varepsilon_{c i}\right)+\sum_{\lambda=1}^{\Lambda} \gamma_{\lambda} \psi_{c i}^{(\lambda)}\right),
$$

где $h$ - постоянная Планка, индексы $c$ и $i$ характеризуют сорт частицы и набор квантовых чисел, определяющих уровень ее внутренней энергии $\varepsilon_{c i}, s_{c i}-$ статистический вес, соответствующий этой энергии, $m_{c}$ и $\mathbf{p}_{c}$ - масса и импульс частицы, $p_{c}^{2} / 2 m_{c}+\varepsilon_{c i}=\psi_{c i}^{(0)}-$ основной скалярный инвариант, соответствующий закону сохранения энергии, $\psi_{c i}^{(\lambda)}(\lambda=\overline{1, \Lambda})-$ аддитивные инварианты столкновений, не зависящие от импульса, $\gamma_{0}, \gamma_{1}, \ldots, \gamma_{\lambda}-$ постоянные величины, которые находятся из условий нормировки:

$$
\begin{gathered}
\sum_{c, i} \int f_{c i}\left(\mathbf{p}_{c}\right)\left(\frac{p_{c}^{2}}{2 m_{c}}+\varepsilon_{c i}\right) d \mathbf{p}_{\mathbf{c}}=\Psi_{0}, \\
\sum_{c, i} \psi_{c i}^{(\lambda)} \int f_{c i}\left(\mathbf{p}_{c}\right) d \mathbf{p}_{c}=\Psi_{\lambda}, \quad \lambda=\overline{1, \Lambda} .
\end{gathered}
$$

Здесь $\Psi_{0}=E$ и $\Psi_{\lambda}-$ суммарные значения полной энергии и инвариантов $\psi_{c i}^{(\lambda)}$ в единице объема.

При квазиклассическом описании поступательных степеней свободы частиц газа, температура которого равна $T$, на каждую такую степень свободы приходится энергия $k T / 2$. В этих условиях

$$
E=\frac{3}{2} n k T+\sum_{c, i} \varepsilon_{c i} n_{c i},
$$

где

$$
n=\sum_{c, i} n_{c i}=\sum_{c, i} \int f_{c i}\left(\mathbf{p}_{c}\right) d \mathbf{p}_{c}
$$


Из соотношений (1), (2) и (4) легко получить равенство

$$
\gamma_{0}=-\frac{1}{k T}
$$

В настоящей работе рассматривается газ, состоящий из частиц одного химического сорта. Предполагается, что температура газа достаточно высока, поэтому его можно считать полностью диссоциированным.

C ростом температуры в газе начинаются процессы ионизации. Гипотетически при достаточно высокой температуре $T$ в газе из химических элементов А (с номером $N$ в периодической системе химических элементов) наряду с нейтральными атомами $A_{0}$ могут присутствовать ионы $A_{c}(c=\overline{1, N})$ с зарядом от +1 до $+N$, а также свободные электроны $e$.

Свободные электроны $e$ и ядра $A_{N}$, лишенные электронной оболочки, обладают лишь поступательной энергией.

Частицы $A_{c}$ сортов $c=\overline{0, N-1}$ имеют внутреннюю энергию $\varepsilon_{c i}$, которая равна сумме энергий $N-c$ электронов, движущихся по некоторым орбитам вокруг ядра.

Пронумеруем эти электроны индексом $l$ в порядке приближения к ядру. Нужно учесть, что каждый такой электрон может вращаться по нескольким орбитам и, следовательно, находиться на разных энергетических уровнях $i_{l}$. Индекс $i$, соответствующий фиксированному уровню энергии частиц сорта $c$, определяется набором квантовых чисел $i_{c+1}, i_{c+2}, \ldots, i_{N}$. Если отсчитывать энергию каждого электрона в оболочке частицы $A_{c}$ от глубины соответствующей потенциальной ямы, то внутренняя энергия $\varepsilon_{c i}$ может быть представлена в виде

$$
\varepsilon_{c i}=\sum_{l=c+1}^{N}\left(\varepsilon_{i_{l}}^{(c)}-I_{l}^{(c)}\right), i_{l}=\overline{0, i_{l}^{*}(c)}, c=\overline{0, N-1},
$$

где $I_{l}^{(c)}$ - потенциал ионизации (энергия, необходимая для отрыва $l$-го электрона от частицы сорта $c$ ), индекс $i_{l}=0$ соответствует невозбужденному состоянию электрона, а $i_{l}^{*}(c)$ - наиболее высокому уровню его энергии.

В ионизованном газе при всех столкновениях сохраняется также общее число ядер и электронов. Поэтому существует еще два скалярных аддитивных инварианта $\psi_{c i}^{(1)}$ и $\psi_{c i}^{(2)}$, где индексы 1 и 2 соответствуют числам ядер и электронов в рассматриваемой частице. Следовательно, будем иметь

$$
\begin{gathered}
\psi_{0, i}^{(1)}=1, \quad \psi_{1, i}^{(1)}=1, \ldots, \quad \psi_{N-1, i}^{(1)}=1, \\
\psi_{N, i}^{(1)}=1, \quad \psi_{e, i}^{(1)}=0, \\
\psi_{0, i}^{(2)}=N, \quad \psi_{1, i}^{(2)}=N-1, \ldots, \quad \psi_{N-1, i}^{(2)}=1, \\
\psi_{N, i}^{(2)}=0, \quad \psi_{e, i}^{(2)}=1 .
\end{gathered}
$$

Таким образом, равновесные функции распределения (1) в пространственно-однородном одноатомном термически ионизованном газе могут быть с учетом равенства (5) и соотношений (7), (8) представлены в виде

$$
\begin{gathered}
f_{c i}\left(\mathbf{p}_{c}\right)=\frac{s_{c i}}{h^{3}} \exp \left(-\frac{p_{c}^{2} / 2 m_{c}+\varepsilon_{c i}}{k T}+\gamma_{1}+\gamma_{2}(N-c)\right) \\
c=\overline{0, N-1}, \quad i=\overline{i_{c+1}, i_{N}}, \quad i_{l}=\overline{0, i_{l}^{*}(c)} \\
f_{N}\left(\mathbf{p}_{N}\right)=\frac{1}{h^{3}} \exp \left(-\frac{p_{N}^{2} / 2 m_{N}}{k T}+\gamma_{1}\right) \\
f_{e}\left(\mathbf{p}_{e}\right)=\frac{1}{h^{3}} \exp \left(-\frac{p_{e}^{2} / 2 m_{e}}{k T}+\gamma_{2}\right)
\end{gathered}
$$

\section{2. Равновесный состав термически ионизованного газа}

Для определения равновесных концентраций тяжелых частиц $n_{c}(c=\overline{0, N})$ и свободных электронов $n_{e}$ функции (9)-(11) нужно проинтегрировать по пространству импульсов, а функции (9) еще и просуммировать по уровням электронной энергии. В результате будем иметь

$$
n_{c}=\left\{\begin{array}{l}
Z_{c}(T) \exp \left(\gamma_{1}+\gamma_{2}(N-c)\right), \quad c=\overline{0, N-1}, \\
Z_{N}(T) \exp \left(\gamma_{1}\right), \\
Z_{e}(T) \exp \left(\gamma_{2}\right) .
\end{array}\right.
$$

где $Z_{c}(T)$ - статистические суммы, которые задаются следующими выражениями:

$$
\begin{aligned}
Z_{c}(T)=\left(\frac{2 \pi m_{c} k T}{h^{2}}\right)^{\frac{3}{2}} & \prod_{l=c+1}^{N} \sum_{i_{l}} s_{i_{l}} \exp \frac{\varepsilon_{i_{l}}^{(c)}-I_{l}^{(c)}}{k T}, \\
c & =\overline{0, N-1}, \\
Z_{N}(T) & =\left(\frac{2 \pi m_{N} k T}{h^{2}}\right)^{\frac{3}{2}}, \\
Z_{e}(T) & =\left(\frac{2 \pi m_{e} k T}{h^{2}}\right)^{\frac{3}{2}} .
\end{aligned}
$$

Чтобы вычислить статистические суммы $Z_{c}(T)$ по формулам (13), необходимо знать такие энергетические характеристики частиц, как уровни электронной энергии $\varepsilon_{i_{l}}^{(c)}$ и значения потенциалов ионизации $I_{l}^{(c)}$. Их можно найти в различной справочной литературе и открытых базах данных, например, [10].

Если равновесная температура $T$ известна, то для определения равновесного состава остается только рассчитать коэффициенты $\gamma_{1}$ и $\gamma_{2}$ в формулах $(12)$, используя условия нормировки (3), которые в рассматриваемых условиях соответствуют сохранению общего числа ядер и электронов (имеются в виду как связанные, так и свободные ядра и электроны).

Если начальная числовая плотность атомов равна $n^{(0)}$, то соответствующие условия сохранения имеют вид

$$
\sum_{c=0}^{N} Z_{c}(T) \exp \left(\gamma_{1}+\gamma_{2}(N-c)\right)=n^{(0)}
$$




$$
\begin{aligned}
& \sum_{c=0}^{N-1} Z_{c}(T) \exp \left(\gamma_{1}+\gamma_{2}(N-c)\right)(N-c) \\
& +Z_{e}(T) \exp \left(\gamma_{2}\right)=n^{(0)} N .
\end{aligned}
$$

Замечание. Легко видеть, что равновесные концентрации (12) тождественно удовлетворяют уравнениям Саха, записанным для процессов ионизации-нейтрализации и обмена электронами при столкновениях. При этом из уравнений (14) следуют условия сохранения массы и электронейтральности.

Если ввести обозначения

$$
e^{\gamma_{1}}=x, \quad e^{\gamma_{2}}=y,
$$

то равновесные концентрации можно представить следующим образом

$$
n_{c}=\left\{\begin{array}{l}
Z_{c}(T) x y^{N-c}, \quad c=\overline{0, N} \\
Z_{e}(T) y
\end{array}\right.
$$

При этом уравнения системы (14) примут вид

$$
\begin{gathered}
\sum_{c=0}^{N} Z_{c}(T) x y^{N-c}=n^{(0)}, \\
\sum_{c=0}^{N-1} Z_{c}(T) x y^{N-c}(N-c)+Z_{e}(T) y=n^{(0)} N .
\end{gathered}
$$

Уравнение (17) позволяет выразить $x$ через $y$ :

$$
x=\frac{n^{(0)}}{\sum_{c=0}^{N} Z_{c}(T) y^{N-c}}=\frac{n^{(0)}}{\Phi(y, T)} .
$$

Подставляя (19) в (18), получим уравнение вида

$$
f(y, T)=n^{(0)} N
$$

где

$$
f(y, T)=\frac{n^{(0)}}{\Phi(y, T)} \sum_{c=0}^{N}(N-c) Z_{c}(T) y^{N-c}+Z_{e}(T) y .
$$

Таким образом, использование статистических распределений $(9)-(11)$ и запись равновесных концентраций компонентов ионизованной смеси в виде (16) позволяет свести задачу определения равновесного состава идеальной плазмы к решению одного алгебраического уравнения (20). При этом нам подходит лишь положительное решение этого уравнения.

Из вида формулы (21) следует, что левая часть уравнения (20) равна нулю при $y=0$, а если $y>0$, то $\partial f / \partial y>0$. Поэтому уравнение (20) при любой температуре имеет одно положительное решение.

Уравнение (20) может быть записано в виде

$$
\begin{aligned}
Z_{0} Z_{e} y^{N+1} & +\sum_{c=1}^{N}\left(Z_{c} Z_{e}-n^{(0)}(c-1) Z_{c-1}\right) y^{N+1-c} \\
& -n^{(0)} N Z_{N}=0 .
\end{aligned}
$$

Если из экспериментальных данных заранее известно, что в исследуемом диапазоне температур и давлений в ионизованной смеси могут присутствовать ионы с зарядом не выше $+k(k<N)$, то степень уравнения (22) понизится до $k+1$.

В этих условиях можно считать, что в системе будет сохраняться общее число ионов с зарядом $+k$ и электронов. В смеси будут присутствовать частицы $A_{c}$ $(c=\overline{0, k})$ и свободные электроны. Если не учитывать возбуждение электронных степеней свободы в ионах $A_{k}$, то эти ионы, как и свободные электроны, будут обладать лишь поступательной энергией. При этом каждая из частиц $A_{c}(c=\overline{0, k-1})$ обладает также внутренней энергией $\varepsilon_{c i}$, а индекс $i$ определяется набором квантовых чисел $i_{c+1}, i_{c+2}, \ldots, i_{k}$.

Определение равновесного состава ионизованных газов с однократной или двукратной ионизацией, когда уравнение (22) является квадратным или кубическим и допускает аналитическое решение, описано в работе [11].

В настоящей работе мы приведем результаты исследования равновесного состава термически ионизованной газовой смеси с многократной ионизацией $k \geq 3$, уделяя особое внимание тому влиянию, которое оказывает на степень термической ионизации газа его плотность.

\section{3. Равновесный состав многократно ионизованных газов разной плотности}

Сведение задачи определения равновесного состава термически ионизованной смеси к решению одного алгебраического уравнения особенно важно в ситуации, когда нужно исследовать смеси с многократной ионизацией $k \geq 3$. Однако в этом случае приходится решать уравнение четвертой степени и выше.

Для решения таких уравнений нужно использовать численные методы поиска корней.

Уравнения (22) могут быть представлены в виде

$$
F(y)=0 \text {. }
$$

Для решения таких уравнений может быть применен один из итерационных методов, например метод Ньютона [12]. Как известно, этот метод применим только в том случае, когда функция $F(y)$ дважды непрерывно дифференцируема. Из вида уравнения (22) следует, что это условие выполняется автоматически при любом значении температуры $T$.

В газе с $k$-кратной ионизацией метод Ньютона позволяет найти все $k+1$ корней соответствующего варианта уравнения (22). Далее нам необходимо выбрать вещественный положительный корень. В разд. 2 было доказано его существование и единственность. Подставляя вычисленное значение у в формулу (19), а затем в (16), получим равновесные концентрации компонентов смеси при определенной температуре. Повторяя подобные 


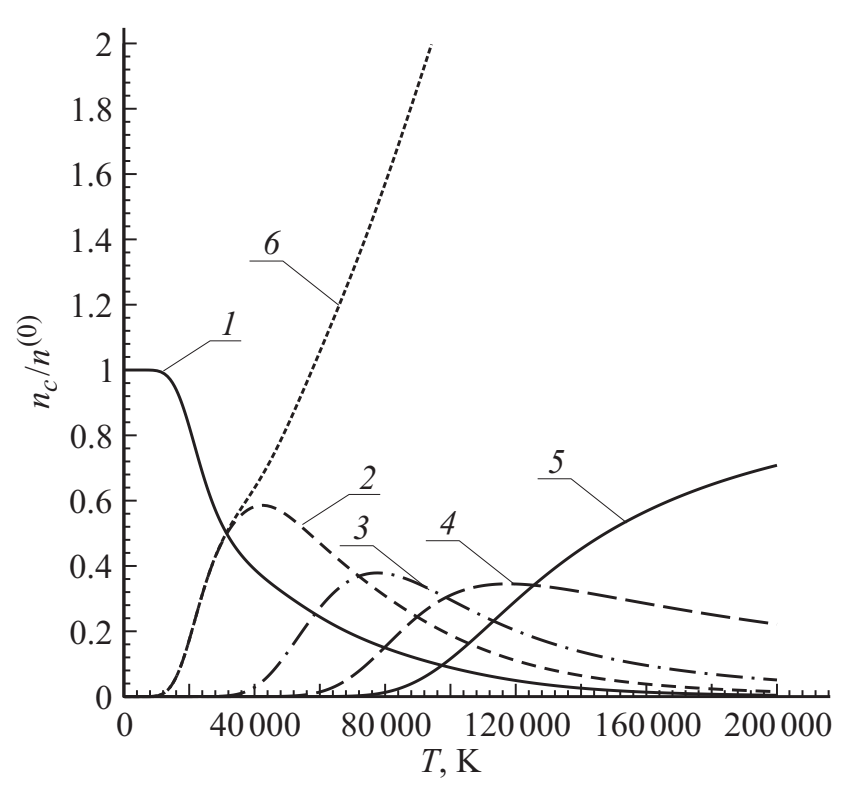

Pис. 1. Относительные концентрации компонентов четырехкратно ионизованного азота. Кривая 1 соответствует $n_{0} / n^{(0)}$, $2-n_{1} / n^{(0)}, 3-n_{2} / n^{(0)}, 4-n_{3} / n^{(0)}, 5-n_{4} / n^{(0)}, 6-n_{e} / n^{(0)}$.

процедуры для разных значений температуры, можем построить температурные зависимости концентраций всех частиц рассматриваемой смеси.

Для иллюстрации приведем результат расчета равновесного состава смеси, в которой могут присутствовать четырехкратно ионизованные атомы азота. Выражения (16) для равновесных концентраций компонентов такой смеси имеют вид

$$
\begin{aligned}
& n_{0}=Z_{0}(T) x y^{4}, \quad n_{1}=Z_{1}(T) x y^{3}, \quad n_{2}=Z_{2}(T) x y^{2} \\
& n_{3}=Z_{3}(T) x y, \quad n_{4}=Z_{4}(T) x, \quad n_{e}=Z_{e}(T) y .
\end{aligned}
$$

На рис. 1 изображены зависимости от температуры равновесных концентраций (23), отнесенных к начальной плотности $n^{(0)}=N_{L}\left(N_{L}\right.$ - число Лошмидта).

Как видно из рис. 1 , при температуре $T \lesssim 12000 \mathrm{~K}$ в равновесной смеси могут присутствать лишь атомы азота $\left(n_{0} / n^{(0)}=1\right)$, затем концентрация атомов монотонно стремится к нулю. На рисунке также можно увидеть последовательно расположенные температурные интервалы, в которых может наблюдаться возрастание относительных концентраций ионов $n_{c} / n^{(0)}(c=1,2,3)$ до некоторых максимумов, а затем их монотонное убывание. Можно проследить, при какой температуре в равновесной смеси с начальной плотностью $n^{(0)}=N_{L}$ могут появиться ионы с зарядом +4 и как их относительная концентрация возрастает с увеличением температуры. Однако для расчета максимума отношения $n_{4} / n^{(0)}$ нужно учитывать возможность пятикратной ионизации атомов азота. При этом на графике хорошо видна кривая, характеризующая монотонное возрастание относительной концентрации свободных электронов $n_{e} / n^{(0)}$.
Из вида уравнений (14) и (20) следует, что равновесный состав ионизованных смесей зависит от их плотности $\rho=m_{0} n^{(0)}$. Эта зависимость была проанализирована на примере расчета равновесных концентраций различных тяжелых частиц $n_{c} / n^{(0)}(c=\overline{0,4})$ и электронов $n_{e} / n^{(0)}$ в ионизованном азоте, начальная числовая плотность которого $n^{(0)}$ менялась в широких пределах.

Результаты этого анализа показаны на рис. 2-7. На каждом из этих рисунков кривая 1 соответствует начальной плотности атомов $n^{(0)}=N_{L}, 2-N_{L} \cdot 10^{-2}$, $3-N_{L} \cdot 10^{-4}, 4-N_{L} \cdot 10^{-6}$.

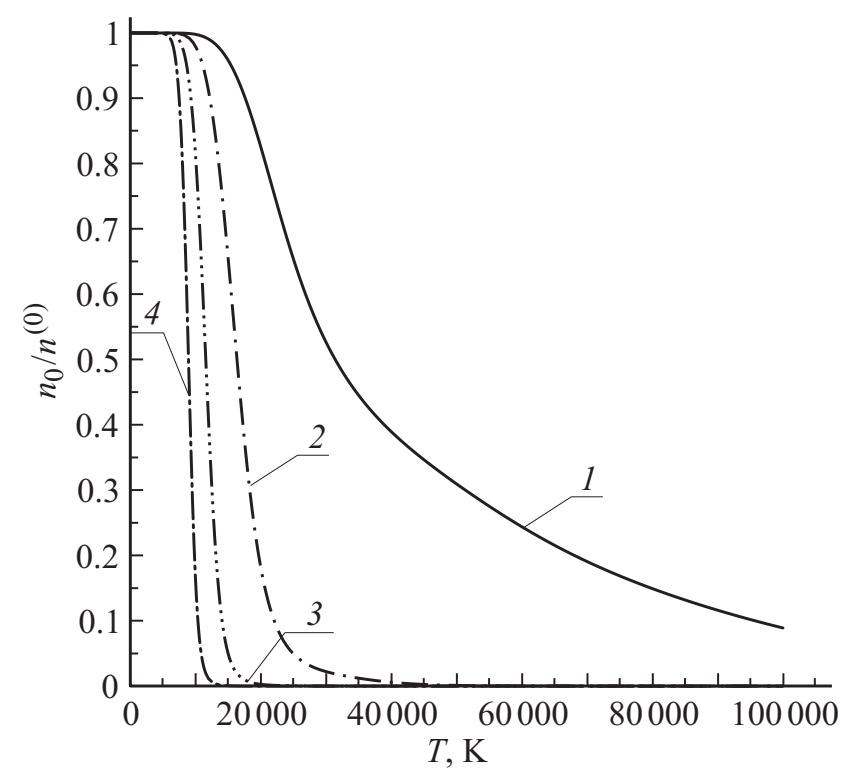

Рис. 2. Температурные зависимости относительных концентрации атомов азота.

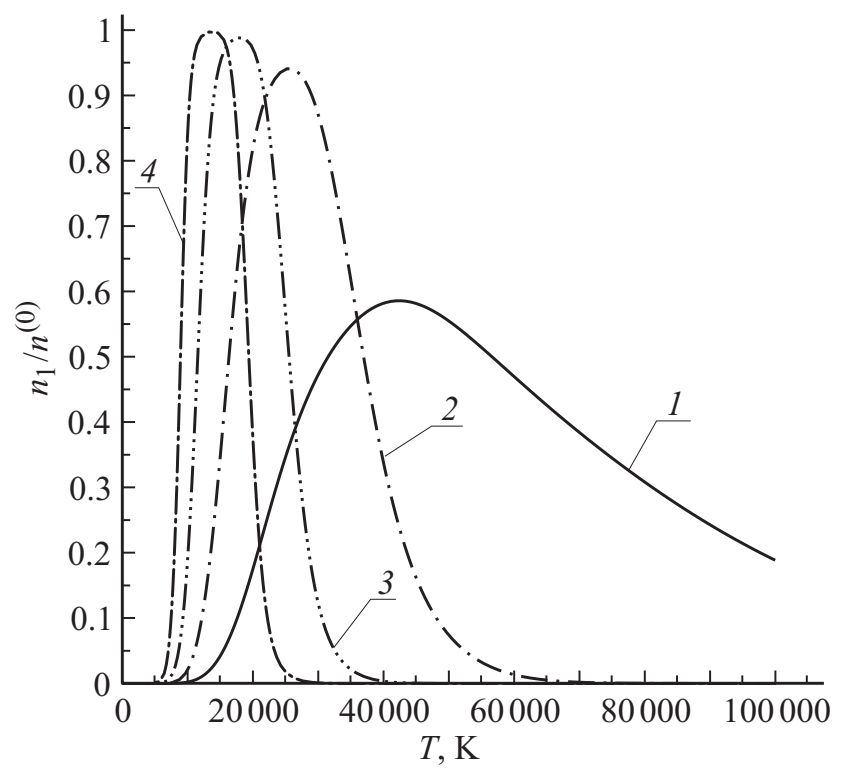

Рис. 3. Температурные зависимости относительных концентрации ионов азота с зарядом +1 . 
Из рис. 2-7 можно видеть, что температуры, при которых в равновесном составе ионизованного азота нужно учитывать присутствие однократно, двукратно, трехкратно и четырехкратно ионизованной фракции и свободных электронов тем ниже, чем более разрежен газ. С физической точки зрения этот вывод можно объяснить тем, что ионизация происходит при парных взаимодействиях частиц, а обратный процесс нейтрализации, как правило, связан с тройными взаимодействиями.

Следует отметить, что предложенный в нашей работе метод исследования равновесного состава термически

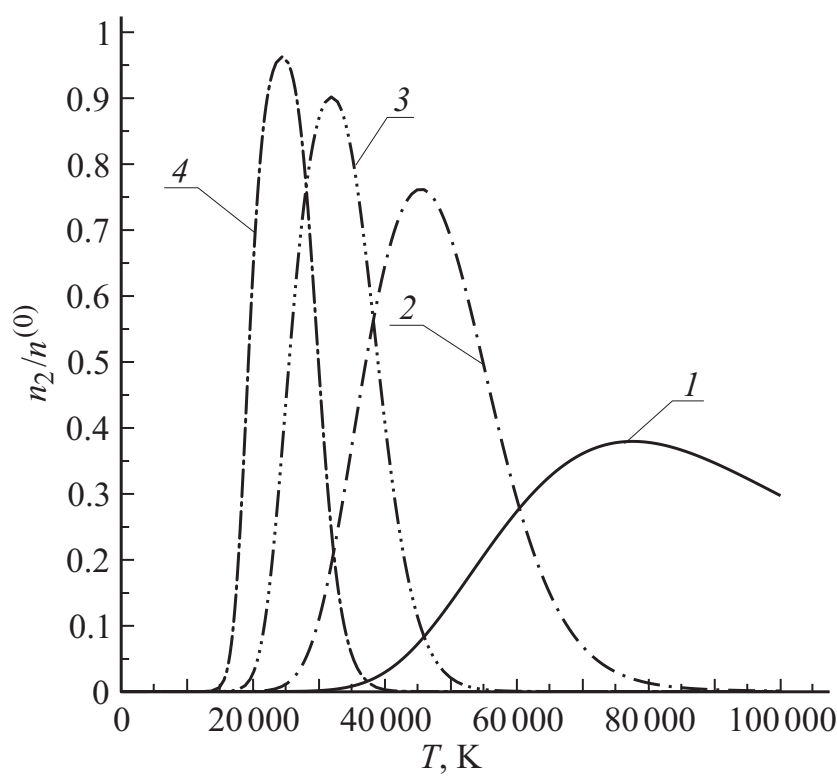

Pис. 4. Температурные зависимости относительных концентраций ионов азота с зарядом +2 .

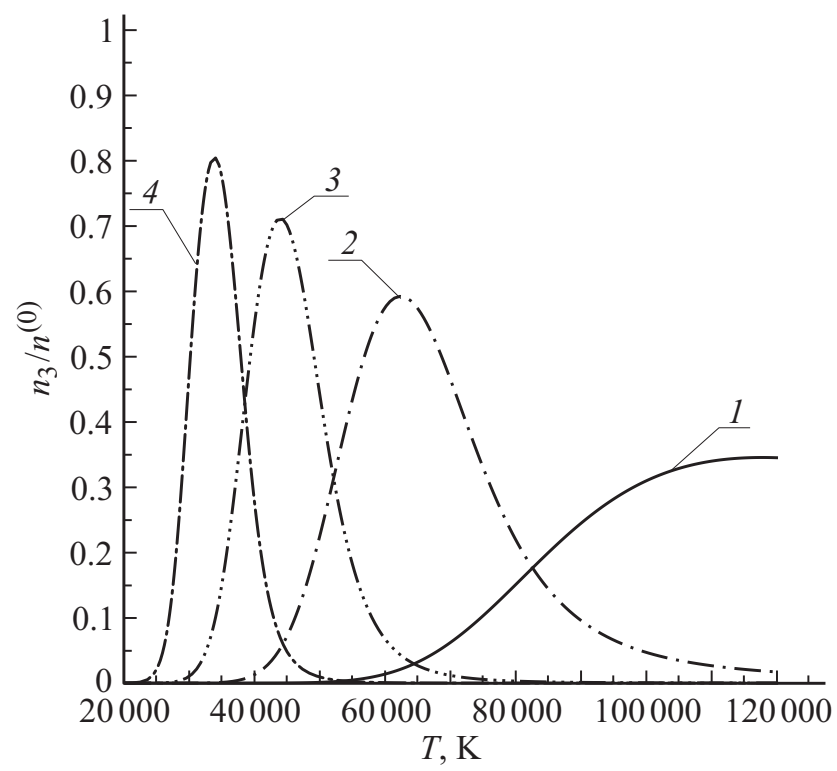

Рис. 5. Температурные зависимости относительных концентраций ионов азота с зарядом +3 .

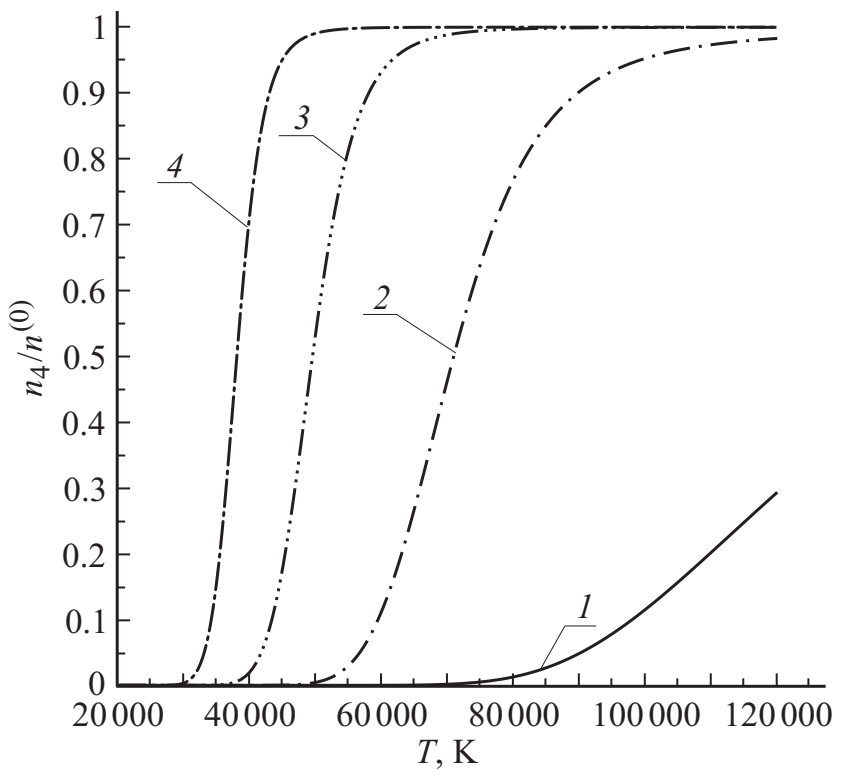

Рис. 6. Температурные зависимости относительных концентраций ионов азота с зарядом +4 .

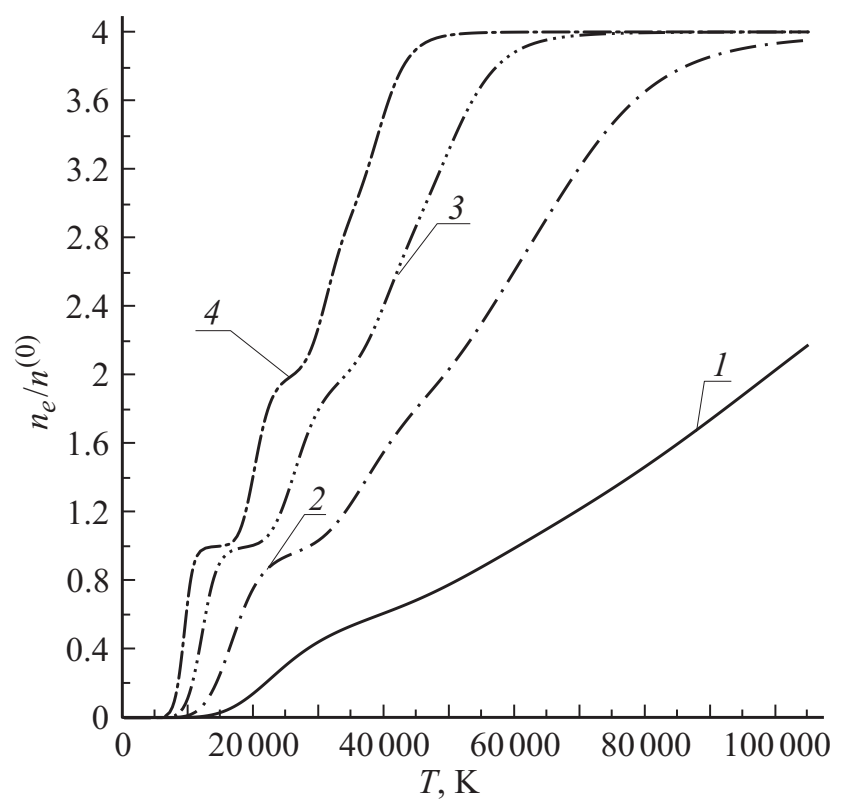

Рис. 7. Температурные зависимости относительных концентраций электронов.

ионизованного одноатомного газа и вид уравнения (20) позволяют еще до проведения расчета предсказать увеличение степени ионизации газа и возрастание равновесных значений относительной концентрации свободных электронов при уменьшении плотности $n^{(0)}$ (для любой фиксированной температуры).

Действительно, относительные концентрации свободных электронов могут быть представлены в виде

$$
\frac{n_{e}}{n^{(0)}}=Z_{e}(T) \tilde{y}, \quad \tilde{y}=\frac{y}{n^{(0)}} .
$$


При этом уравнение (20) запишется следующим образом:

$$
\begin{aligned}
\tilde{f}\left(\tilde{y}, T, n^{(0)}\right)= & \frac{1}{\Phi\left(n^{(0)} \tilde{y}, T\right)} \sum_{c=0}^{N}(N-c) Z_{c}(T)\left(n^{(0)} \tilde{y}\right)^{N-c} \\
& +Z_{e}(T) \tilde{y}=N .
\end{aligned}
$$

В газе определенного сорта при фиксированной температуре левая часть уравнения (25) зависит лишь от $\tilde{y}$ и $n^{(0)}$. При этом соотношение (25) можно рассматривать как неявную функцию

$$
\tilde{f}\left(\tilde{y}, n^{(0)}\right)=N=\mathrm{const}
$$

откуда следует равенство

$$
\frac{d \tilde{y}}{d n^{(0)}}=-\frac{\partial \tilde{f} / \partial n^{(0)}}{\partial \tilde{f} / \partial \tilde{y}} .
$$

Подобно тому, как в разд. 2 было доказано, что $\partial f / \partial y>0$, легко показать справедливость неравенств $\partial \tilde{f} / \partial n^{(0)}>0$ и $\partial \tilde{f} / \partial \tilde{y}>0$.

Таким образом, из равенств (26) и (24) следует возрастание относительной концентрации свободных электронов $n_{e} / n^{(0)}$ при уменьшении $n^{(0)}$ (или плотности газа $\rho)$.

\section{Заключение}

В настоящей работе показано применение наиболее вероятных статистических распределений для расчета равновесного состава термически ионизованных одноатомных газов. Отмечается, что использование этих распределений позволяет избежать решения уравнений Саха, которые удовлетворяются тождественно, а условия сохранения массы и заряда заменяются условиями сохранения общего числа ядер и электронов (14). При этом показано, что можно свести задачу к решению одного алгебраического уравнения (22), степень которого на единицу больше степени ионизации рассматриваемой смеси.

Для случаев однократной и двукратной ионизаций такой подход позволяет получить аналитические формулы, определяющие зависимость равновесных концентраций компонентов ионизованной смеси от температуры и начальной плотности [11].

Исследование равновесного состава многократно ионизованных газов с разной плотностью, проведенное в настоящей работе, позволяет определить температурные зависимости равновесного состава термически ионизованных газов разных сортов.

Показано, что предложенный метод расчета равновесного состава ионизованной смеси позволяет определить влияние ее плотности на температурные зависимости равновесных концентраций ее компонентов и степень термической ионизации. Аналитически доказано, что равновесные значения относительных концентраций ионизованных частиц и свободных электронов тем выше, чем более разреженным является газ. Приведены результаты численного расчета температурных зависимостей равновесных концентраций компонентов четырехкратно ионизованного азота при разных значениях начальной плотности.

Работа выполнена при финансовой поддержке СПбГУ (НИР 6.37.206.2016).

\section{Список литературы}

[1] Энциклопедия по низкотемпературной плазме. / Под ред. В.Е. Фортова. М.: Изд-во Наука, 2000. 633 с.

[2] Энциклопедия по низкотемпературной плазме. Приложение 3-1. / Под ред. В.Е. Фортова, А.Н. Старостина, И.Л. Иосилевского. М.: Изд-во Физматлит, 2004. 495 с.

[3] Mitchner M., Kruger C.H.J. Partially ionized gases. N. Y.: J. Willey and Sons, 1973. 458 p.

[4] Golant V.E., Zilinskij A.P., Sacharov I.E. Fundamentals of plasma physics. N.Y.: J. Willey and Sons, 1980. 528 p.

[5] Жданов В.М. Явления переноса в многокомпонентной плазме. М.: Физматлит, 2009. 299 с.

[6] Capitelli M., Colonna G., DAngola A. Fundamental espects of plasma chemical physics. // N.Y.-Dordrcht-HeidelbergLondon: Springer, 2012.

[7] Ender A.Ya., Kolinsky H., Kuznetsov V.I., Schamel H. // Phys. Report. 2000. Vol. 328. P. 72.

[8] Гиббс Дж. Основные принципы статистической механики. М.: НИЦ Регулярная и хаотическая динамика, 2002. 204 с.

[9] Рыдалевская М.A. Статистические и кинетические модели в физико-химической газодинамике. СПб.: Изд-во СПбГУ, 2003. $248 \mathrm{c}$.

[10] Электронный ресурс. Режим доступа: WEBBOOK.NIST.GOV./CHEMISTRY

[11] Рыдалевская М.А., Романова М.С. Определение равновесного состава ионизованных одноатомных газов // Вест. СПбГУ. 2013. Сер. 1. Вып. 4. С. 108-116.

[12] Березин И.С., Жидков Н.П. Методы вычисления // М.: Изд-во ГИФМЛ, Т. 2. 1959. С. 620. 